Case Report

\title{
Scrub Typhus with Multi Organ Dysfuntion: A Rare Case Report
}

\section{Maj(Dr) Sankar J ${ }^{1}$, Lt Col(Dr) S S Majumdar ${ }^{2}$, Col(Dr) Shyamal Maity ${ }^{3}$, Brig(Dr) Atul khullar ${ }^{4}$, Gp Capt(Dr) Kishore Kumar ${ }^{5}, \operatorname{Capt}\left(\right.$ Dr)Praveen $\mathbf{M}^{6}$}

\author{
${ }^{1}$ Graded specialist(Medicine),Military hospital,Chennai(India) \\ ${ }^{2}$ Graded specialist(Medicine),Military hospital,Chennai(India) \\ ${ }^{3}$ Classified specialist(Anaesthesia and critical care),Military hospital, Chennai(India) \\ ${ }^{4}$ Commandant ,Military hospital, Chennai(India) \\ ${ }^{5}$ Senior Advisor(Medicine and Oncology), Command hospital ,Banglore(India) \\ ${ }^{6}$ Medical officer,Military Hospital, Chennai(India)
}

Corresponding author: Maj(Dr) Sankar J

Graded specialist(Medicine) ,Military hospital ,Ekkatuthangal ,Chennai-600032.India

\section{INTRODUCTION}

Scrub typhus is a rickettsial disease caused by O.tsutsugamushi which is maintained by trans-ovarian transmission in trobiculid mite ${ }^{1}$. It causes a disseminated vasculitic and perivascular inflammatory lesions resulting in significant vascular leakage and end organ injury.The presentation can vary from self limiting illness to deadly systemic complications like ARDS, pneumonitis, myocarditis,AKI, Hepatic Dysfuntion and neurological manifestations ${ }^{2}$.The classical clinical presentation includes an eschar,maculopapular rash and regional lymphadenopathy which are very rare. Incubation period is 6-21 days. It is important to differentiate it from other tropical fevers. We are reporting a case of scrub typhus from Vellore district of Tamilnadu with multi organ dysfunction in form of ARDS, Myocarditis,hepatic dysfunction and neurological manifestations and finally recovered after prolonged ventilator support.

\section{CASE PRESENTATION}

70 year old female without any previous co morbidities, a resident of Vellore district of Tamilnadu reported to a local hospital at Vellore with the history of fever of 5 days duration. She was given short course of antibiotics, but the fever persisted. From 6th day of illness, she developed breathlessness and brought to Military hospital Chennai. Clinical examination revealed pulse-114/min regular, BP118/76 mm Hg,RR-26/min ,spo2-86\% at room air. Respiratory system examination -bilateral crepitations present Rt>Lt. Eschar was present on the left shoulder with Lt axillary lymphadenopathy. Investigations revealed $\mathrm{Hb}-7.2 \mathrm{gm} / \mathrm{dl}$, PBS-Neutrophilic leukocytosis, thrombocytopenia. Serum bilirubin- $\quad 4.3 \mathrm{~g} / \mathrm{dl}$.ALT/AST/ALP-75/166/868 IU/L.Renal functions were normal. Sodium/potassium-130/3.7.Chest X ray PA view-revealed patchy opacities in both lung fields suggestive of early ARDS.2 D Echocardiography was normal on admission .Scrub typhus IGM was positive. Other fever work up was negative. Arterial blood gas analysis revealed Type 1 respiratory failure. She was started on Non Invasive ventilatory support, Parentral Azithromycin, doxycycline and other supportive measures. On third day of admission, she had altered sensorium and worsening respiratory distresss,in view of which she was put on mechanical ventilator support. On the seventh day of admission, she had asymmetric quadriparesis .Clinically we considered hypokalemia or GBS or critical illness neuropathy as the possibility. Her serum electrolytes were normal.CSF analysis was done and repeated after 1 week showed albumino cytological dissociation .MRI brain and spine was normal. Nerve conduction study revealed sensory motor neuropathy in both lower limbs both axonal and demylinating.Over the course she continued to have fever ,sensorium worsened, progressive hepatic dysfunction .Her antibiotic cover was upgraded,rifampicin was added and continued on ventilator support and intravenous immunoglobulin was given for 5 days.On day 15 tracheostomy was done in view of anticipated prolonged mechanical ventilation. On day 17 she had sudden onset of respiratory distress, hypotension.Chest examination revealed bilateral coarse crepitations.ECG showed ST-T changes and cardiac enzymes was positive.2DEchocardography revealed dilated all four chambers, global hyokinesia with EF-32\%.She had myocarditis and managed with ionotropic support and diuretics .She became afebrile and was continued on ventilatory support,ionotropic support,broad spectrum antibiotics and total parentral nutrition.Gradually her sensorium improved, ionotropic support was tapered off.Repeated weaning trials was unsuccessful due to ventilator associated pneumonia. Gradually she improved clinically and radiologically, her hepatic functions improved and finally extubated after 40 days of ventilator support. Tracheostomy was closed and was discharged on medication with advice of 
regular follow up.

\section{DISCUSSION}

Scrub typhus is a rickettsial disease caused by bite of infected larval mite belonging to the family Trombiculidae,genus and sub genus leptotrombidium.An estimation of one million scrub typhus cases occur annually over the world ${ }^{3}$.It is endemic to a part of the world knows as tsutsugamushi triangle from northern Japan in the north to Australia in the south and to Pakistan in the west. ${ }^{4}$ Increase in the prevalence has been reported from Asian countries, may be due to urbanisation in rural areas and wide spread use of antimicrobials ${ }^{5}$. A lot of cases are reported from cooler climates of south india ${ }^{6}$,and many are unreported.It is considered as a re emerging disease in India ${ }^{7}$.It is grossly underdiagnosed in india due to lack of specific tests,limited awareness and low suspicion rate among clinicians. Our case was reported from Vellore district in Tamilnadu,and diagnosed only in second week of illness due to late presentation.

We encountered a spectrum of complications during the management like Acute respiratory distress syndrome, myocarditis, hepatic dysfunction of which the most interesting was the neurological complications like encephalitis and quadriparesis in the second week likely to be post infectious GBS based on the supportive evidence of CSF analysis and nerve conduction studies.Literature revealed that neurological complications ${ }^{8}$ do occur in scrub typhus of which a few cases of post infectious GBS following scrub typhus ${ }^{9}$ are reported.MRI study of brain and spine was normal and there was no serological evidence of EBV,CMV or HIV.It was managed with intravenous immunoglobulin $2 \mathrm{~g} / \mathrm{kg}$ for 5 days along with other supportive measures.Her upper limb power improved and at the time of discharge, she had some residual paraparesis.

The cheapest test to diagnose scrub typhus is weil-felix test which lacks sensitivity and specificity. The gold standard is the immunoflurescence antibody tests(IFA).Quantitative enzyme linked immunosorbent assay(ELISA) for the detection of IGM antibodies is now commercially available which was used in our patient.

Doxycycline is the drug of choice and duration is 7-14days.In small children and pregnant woman azithromycin is the drug of choice.In our case, patient responded to a combination of azithromycin,doxycycline and rifampicin,indicating resistance to doxycyline monotherapy.A combination therapy with doxycycline and rifampicin should be used in areas where there is poor response to doxycycline alone. ${ }^{10}$

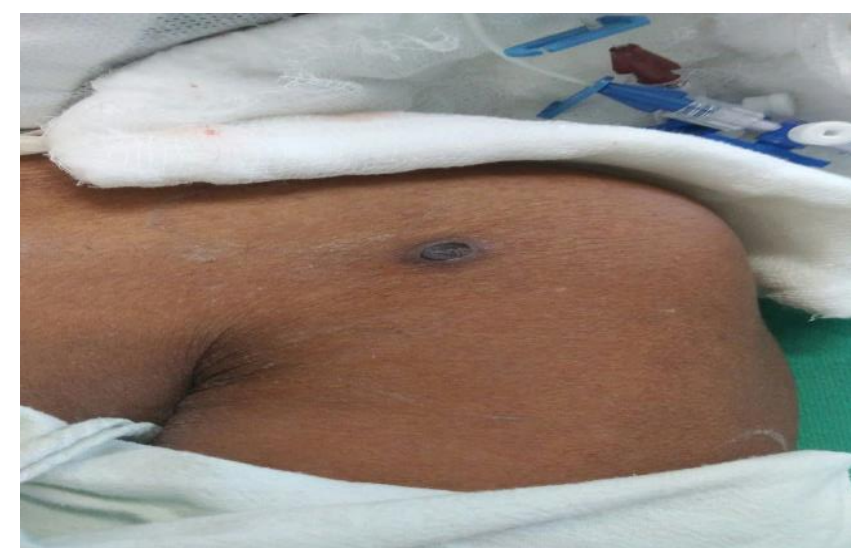

\section{ESCHAR OF SCRUB TYPHUS ON LT SHOULDER}

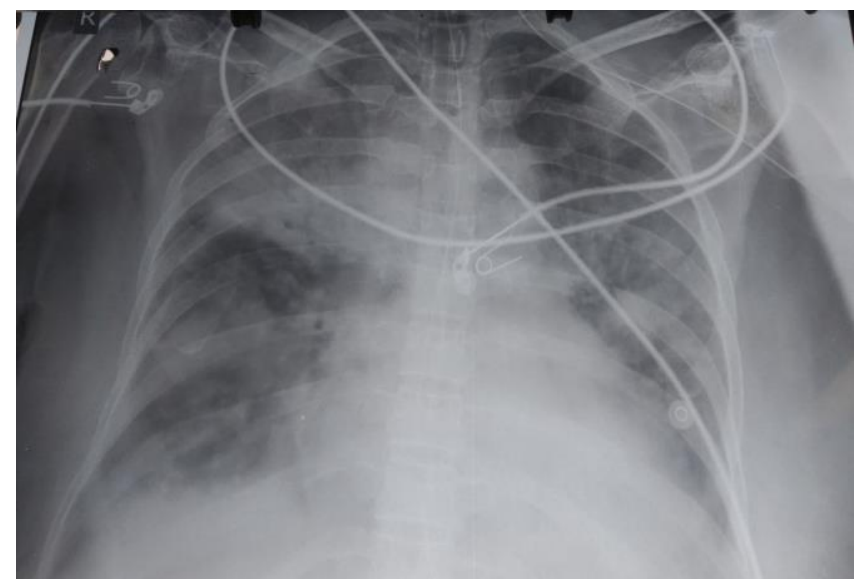

\section{ACUTE RESPIRATORY DISTRESS SYNDROME IN SCRUB TYPHUS.}

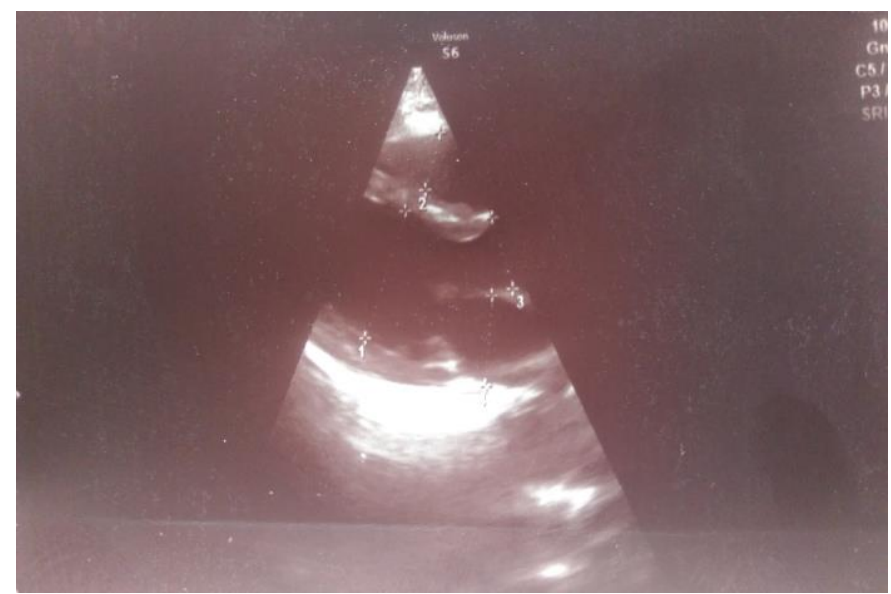

\section{D ECHO CARDIOGRAPHY SHOWING DILATED ALL FOUR CARDIOMYOPATHY) \\ CONCLUSION}

Scrub typhus remains undiagnosed due to non specific presentation and lack of specific laboratory tests.It usually presents as a short febrile illness, but can lead to wide array of systemic complications like Pneumonitis,ARDS,Hepatic Dysfuntion,myocarditis and neurological manifestations.It should be kept in the differential diagnosis of tropical fever and one should be deligent in looking for an Eschar,as early diagnosis can drastically reduce the mortality and morbidity.

\section{REFERENCES}


[1] David H.Walker,J.Stephen Dumler,Thomas Marrie northern thialand.Lancet.1996;348(9020);86-89. ;Rickettsial Diseases; Harrison 's principles of internal medicine 1154.

[2] P B Meena kumara,Sravanthi Devi Reddy;A case report of scrub typhus with multi organ dysfunction;kerala medical journal july-september 2015 volume V111 Issue 3.

[3] Silpapojakul K.Scrub typhus in the Western Pacific Region .Ann Acad Med singapore,26:794-800,1997.

[4] Mc Crumb FR,stockyard JL,Robinson CR,etal.Leptospirosis in Malaya.I.Sporadic cases among military and civilian personnel.Am J Trop Med Hyg. 1957;6(2)238-56.

[5] Seomg SY,choi MS,Kim I.Orienta tsutsugamushi infection:over view and immune responses.Microbes Infect.2001;3(1):11-21.

[6] Mathai E,Rolain JM,Verghese GM,etal.Outbreak of scrub typhus in southern India during the cooler months.Ann N Y Acad Sci.2003;990:359-364.

[7] Padbiri V S,Gupta N P.Rickettsiosis in india:R Review .J Indian Med SSOC,71:104-107,1978.

[8] Silpapojakul K,Ukkachoke C,Krisanapan S.Rickettsial meningitis and encephalitis.Arch Intern Med 1991;151:1753-1757.

[9] Ju IN,etal.Guillian-Barre syndrome after scrub typhus infection ; The Korean Journal of Internal Medicine Vol.26,No.4,December 2011.

[10] Watt G,Chouriyagune C,Ruangweerayud R,etal.Scrub typhus infections poorly responsive to antibiotics in 